\title{
Neoadjuvant Radiotherapy in Stage I Cancer of the Lower Rectum
}

\author{
Antonio Jose Tiburcio Alves Junior ${ }^{1 *}$, Gustavo Alejandro Gutierrez Espinoza', \\ Luciane Hiane Oliveira'2, Sergio Oliva Banci², Joaquim Simoes Neto ${ }^{3}$, \\ Odorino Hideyoshi Kagohara ${ }^{3}$, Jose Alfredo Reis Junior ${ }^{3}$, Jose Alfredo Reis Neto ${ }^{4}$ \\ ${ }^{1}$ Coloproctology Department, Clínica Reis Neto, Campinas, Brazil \\ ${ }^{2}$ Coloproctology Department, Clinica Reis Neto, Catholic University Campinas, Campinas, Brazil \\ ${ }^{3}$ Surgery Department, Clinica Reis Neto, Catholic University Campinas, Campinas, Brazil \\ ${ }^{4}$ Surgery Department, Catholic University of Campinas, Campinas, Brazil \\ Email: jareisneto@msn.com
}

Received 12 February 2014; revised 10 March 2014; accepted 17 March 2014

Copyright (C) 2014 by authors and Scientific Research Publishing Inc.

This work is licensed under the Creative Commons Attribution International License (CC BY). http://creativecommons.org/licenses/by/4.0/

c) (i)

Open Access

\begin{abstract}
Introduction: The mortality rate in cancer of the lower rectum is related to the incidence of local recurrence, in the first 5 years. For stage I tumors, local excision has being increasingly used, but recent studies showed a higher incidence rate of local recurrence. Therefore, preoperative radiotherapy should be considered even for these tumors, as an attempt to prevent recurrence and provide cure. Objective: To show the effectiveness of neoadjuvant radiotherapy in stage I cancer of the lower rectum of a cohort population. Materials and Method: A cohort study in a prospective database was made with a total of 75 patients considered as stage I cancer of the lower rectum. Preoperative long course of 4500 cG radiotherapy was performed in this selected group of patients and followed up for a minimum period of five years. Results: Stage I/TI group had 27 patients. All of them presented complete response to the treatment and did not need to be submitted to surgery. Five years follow up with no recurrence. The stage I/TII group had 48 patients. After neoadjuvant radiotherapy, 8 patients had to be submitted to surgery for persistent tumor. All were submitted to full total local excision (FTLE), but anatomopathological examination showed no residual cancer. Conclusion: Preoperative long course of 4500 cG irradiation, not only reduced the local recurrence and mortality rate in lower rectal cancer, but also reduced indication for surgery in patients with stage I cancer of the lower rectum.
\end{abstract}

\section{Keywords}

Rectal Cancer, Cancer of the Lower Rectum, Irradiation, Preoperative Radiotherapy, Local Recurrence, Mortality, Survival

"Corresponding author. 


\section{Introduction}

Preoperative radiotherapy in cancer of the lower rectum has been used since 1975 [1]. The surgical management currently includes a broad spectrum of operative procedures ranging from radical operations to innovative sphincter-preserving techniques; new and improved radiation techniques emerged (conformal radiotherapy, intraoperative radiotherapy) with or without combinations of chemotherapies [2]-[5].

The mortality rate is related to local recurrence and distant metastasis [6]. Therefore, adequate surgical technique is mandatory in the treatment of the rectal cancer [6] [7]. It is also important to consider prognostic factors such as the pathologic $\mathrm{T}$ (tumor) and $\mathrm{N}$ (nodal) classification, circumferential resection margin, and response to neoadjuvant therapy [3]. Nodes can be positive even in early tumors [8].

For stage I rectal cancer, local excision has being used increasingly, but recent studies showed the need for caution with the use of this technique, as it does not consider the possibility of a positive node in such tumors [3] [7] [8].

Nowadays, appropriate staging plays an increasingly important role, because many treatment decisions must be based on preoperative staging [2] [3].

Current guidelines advocate for neoadjuvant treatment for stage II and an III tumor, once it has been proved that neoadjuvant radiation reduces local recurrence risk and improves long-term survival [9]-[12].

However, knowing that preoperative radiotherapy is able to decrease, significantly, the number of undifferentiated cells; diminish the grade of tumor invasion in the rectal wall; reduce, statistically, the incidence of local recurrence and alter long-term survival rate, leads us to believe that neoadjuvant therapy should be used in early tumors as well [1].

\section{Objectives}

To analyze the results of neoadjuvant radiotherapy in stage I cancer of the lower rectum of a cohort population.

\section{Methods}

A cohort study in a prospective database was made from 1978 to 2012, with a total of 75 patients with lower rectum cancer considered as stage I cancer. These individuals were submitted to preoperative radiotherapy. There were 27 patients stage I/TI and 48 patients stage I/T2. All of them had lower rectum adenocarcinoma and were followed by a 5 -year minimum.

There was no gender, race and age distinction.

Preoperative dosage of CEA, gamma GT, colonoscopy and abdominal ultrasound were performed in all the patients to stage the tumor. When available endorectal ultrasound was performed to evaluate size and infiltration of the tumor before and after the irradiation.

Proctoscopy and digital examination were performed at diagnosis and after the end of the irradiation treatment to evaluate tumor extension and wall infiltration.

Preoperative radiotherapy was performed with $200 \mathrm{cGy} /$ daily for 4 consecutive weeks up to a total of 4500 cGy, by means of a Linear Megavoltage Accelerator (25 MeV), in anterior and posterior pelvic fields.

Post-radiation protocol included periodical examination every 3 months for the first two years, with digital examination (or careful perineal palpation) and evaluation of the CEA and every 6 months for the next consecutive three years. Abdominal ultrasound was performed yearly and colonoscopy every other year. When clinical assessment suggested local or general recurrence, a CT scan or MR was accomplished.

\section{Results}

The 75 patients with lower rectum cancer were followed for a minimum period of 5 years.

The stage I/TI group had 27 patients, all of them submitted to the same protocol of neoadjuvant therapy. Everyone presented complete response to the treatment and did not need to be operated. During the follow-up time of five years, this group showed no recurrence rate.

The stage I/TII group had 48 patients, all of them submitted to the same protocol of neoadjuvant therapy. During the follow-up, 8 patients had to be operated due to remaining lesion or scar. They were submitted to full total local excision. After evaluating the pathological specimen, none of them proved to be adenocarcinoma. It was found dysplasia and adenoma (Figure 1). 
One patient of this group had a distant metastasis in the lungs after 5 years (Figure 2).

The other 40 patients had complete response after neoadjuvant radiotherapy.

\section{Discussion}

Treatment of rectal cancer has dramatically evolved during the last three decades shifting toward a tailored approach based on preoperative staging and response to neoadjuvant therapy [13].

Preoperative radiotherapy reduces the risk of local recurrence in patients with operable rectal cancer, especially local recurrence, which is responsible for the great majority of deaths in the first two years after surgery [14].

Previous studies showed that there is a significant difference in the five-year survival rates in patients that received preoperative radiotherapy, who had a corrected survival rate of 80 percent versus 34.4 percent of nonirradiated patients. The local recurrence rate was 2.9 percent in patients that received neoadjuvant therapy versus 23.5 percent in those who had not [1].

Endorectal ultrasound (ERUS) displays accuracy of 71\% - 91\% and 69\% - 97\% for T stage I and II respectively, and 62\% - 83\% for nodal staging [15]. Results confirmed in 200 patients that after the neoadjuvant irradiation a change of cellular differentiation and an involution of the tumor size and volume occurred [1] [15]-[18].

According to Aguilar, patients undergoing local resection presented recurrence rate of 18\% (T1) and 37\% (T2) with 54 months of follow-up.

Paty et al. showed recurrence rates of $17 \%$ to $74 \%$ for T1 rectal cancers and $26 \%$ to $72 \%$ for T2 cancers with a median time to relapse of 1.4 years (range $0.4-7.0$ ).

Patients undergoing local excision with transanal endoscopic microsurgery presented recurrence of 13\% (T1), and 17\% (T2) with follow-up of 24 months [19].

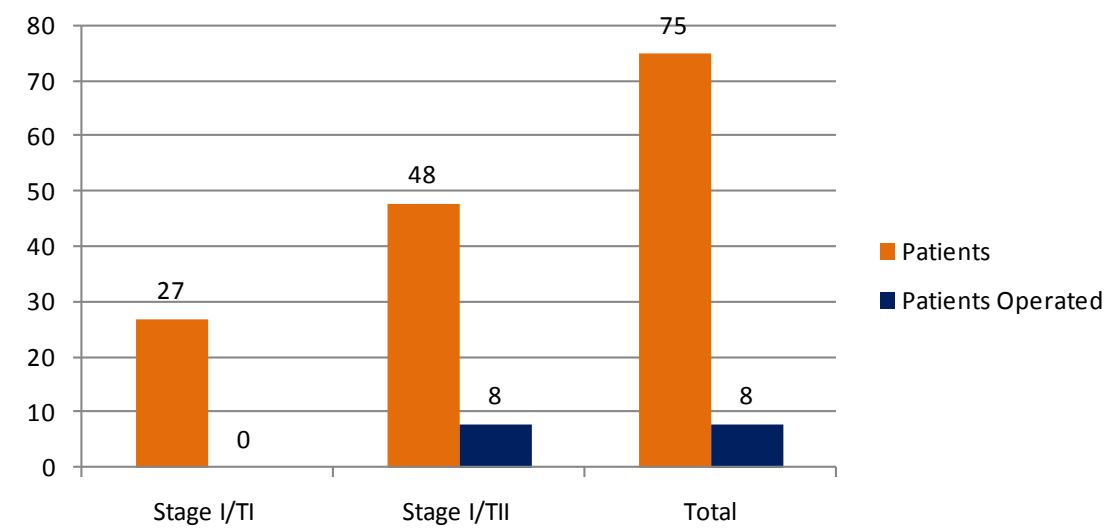

Figure 1. Stage I rectal cancer patients submitted to neoadjuvant therapy and operated.

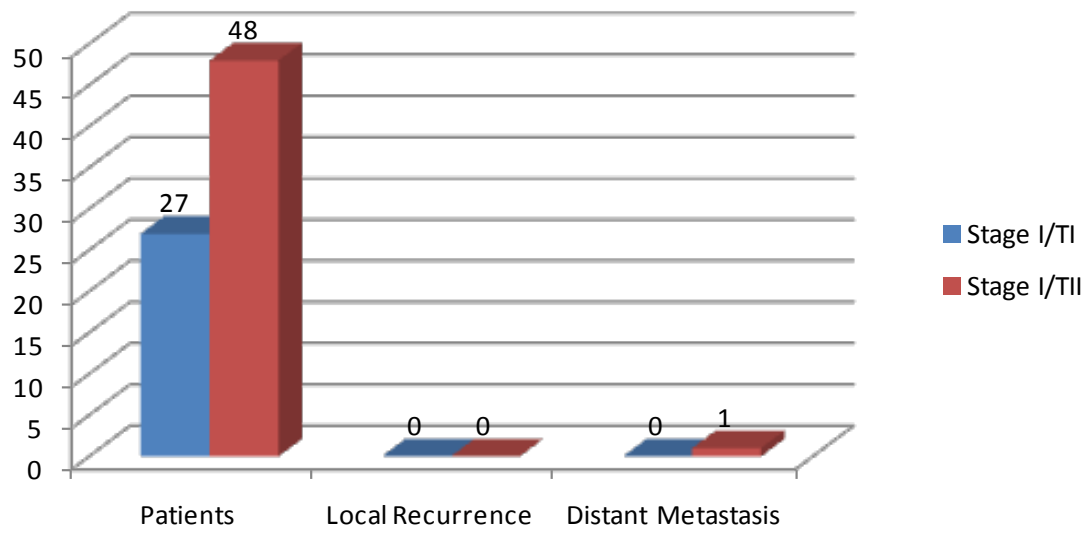

Figure 2. Local recurrence and distant metastasis after 5 years follow-up. 
Table 1. Risk of local recurrence based on the proposed treatment.

\begin{tabular}{cccc}
\hline Local recurrence & $\mathrm{T} 1$ & $\mathrm{~T} 2$ & Surgery \\
\hline Aguilar (2000) & $18 \%$ & $37 \%$ & LR \\
Paty (2002) & $17 \%-74 \%$ & $26 \%-76 \%$ & LR \\
Stipa (2004) & $13 \%$ & $17 \%$ & TEM \\
Bebenek (2009) & $4.4 \%$ & $4.4 \%$ & APR \\
Lezoch (2002) & ---- & $2.85 \%$ & RDT + TEM \\
Reis Neto (2012) & ----- & $2.6 \%$ & RDT + SUR \\
\hline
\end{tabular}

Bebenek showed 4.4\% recurrence rate for T1 and T2 in two years follow-up after abdominoperineal resection [20].

On the other hand, Lezoch showed a recurrence rate similar to the one presented in this study, with no recurrence in $\mathrm{T} 1$ and $2.85 \%$ in $\mathrm{T} 2$. The similar results are due to the use of neoadjuvant radiotherapy before transanal endoscopic microsurgery [21] [22].

Those results showed that receiving neoadjuvant therapy carries less risk of local recurrence than surgical techniques that did not receive (local excision, transanal endoscopic microsurgery, abdominoperineal resection, low anterior resection) (Table 1).

This study did not contemplate the possible complications presented by radiotherapy or alluded to molecular analysis since this was not its goal [23]-[30].

\section{Conclusion}

In this cohort, the use of neoadjuvant long course of 4500 cG radiotherapy reduced the risk of local recurrence, the mortality rate, and the need of surgery in stage I cancer of the lower rectum.

\section{References}

[1] Reis Neto, J.A., Reis Jr., J.A., Kagohara, O., Simoes Neto, J., Banci, S.O. and Oliveira, L.H. (2012) Adjuvant Therapy on Cancer of the Lower Rectum. Evaluation of the Effects of Preoperative Radiotherapy on the Prognosis of Patients with Cancer of the Lower Rectum. Journal of Cancer Therapy, 3, 912-919. http://dx.doi.org/10.4236/jct.2012.326117

[2] Fichera, A. and Allaix, M.E. (2013) Paradigm-Shifting New Evidence for Treatment of Rectal Cancer. Journal of Gastrointestinal Surgery, 18, 391-397.

[3] Das, P. and Crane, C.H. (2009) Staging, Prognostic Factors, and Therapy of Localized Rectal Cancer. Current Oncology Reports, 11, 167-174. http://dx.doi.org/10.1007/s11912-009-0025-3

[4] Kachnic, L.A. (2007) Adjuvant Chemoradiation for Localized Rectal Cancer: Current Trends and Future Directions. Gastrointestinal Cancer Research, 1, 64-72.

[5] Sauer, R. (2002) Adjuvant and Neoadjuvant Radiotherapy and Concurrent Radiochemotherapy for Rectal Cancer. Pathology \& Oncology Research, 8, 7-17. http://dx.doi.org/10.1007/BF03033695

[6] Wagner, T.D., Fakih, M.G. and Yang, G.Y. (2010) Management of Stage II/III Rectal Cancer. Journal of Gastrointestinal Oncology, 1, 112-119.

[7] Sauer, R. and Rödel, C. (2002) Adjuvant and Neoadjuvant Radiochemotherapy of Rectal Carcinoma. Praxis, 91, 14761484. http://dx.doi.org/10.1024/0369-8394.91.37.1476

[8] Pucciarelli, S., Capirci, C., Emanuele, U., Toppan, P., Friso, M.L., Pennelli, G.M., Crepaldi, G., Pasetto, L., Nitti, D. and Lise, M. (2005) Relationship between Pathologic T-Stage and Nodal Metastasis after Preoperative Chemoradiotherapy for Locally Advanced Rectal Cancer. Annals of Surgical Oncology, 12, 111-116. http://dx.doi.org/10.1245/ASO.2005.03.044

[9] Loos, M., Quentmeier, P., Schuster, T., Nitsche, U., Gertler, R., Keerl, A., Kocher, T., Friess, H. and Rosenberg, R. (2013) Effect of Preoperative Radio (Chemo) Therapy on Long-Term Functional Outcome in Rectal Cancer Patients: A Systematic Review and Meta-Analysis. Annals of Surgical Oncology, 20, 1816-1828. http://dx.doi.org/10.1245/s10434-012-2827-z

[10] Kulu, Y., Ulrich, A. and Büchler, M.W. (2012) Resectable Rectal Cancer: Which Patient Does Not Need Preoperative Radiotherapy? Digestive Disease, 30, 118-125. http://dx.doi.org/10.1159/000342040

[11] Rödel, C., Trojan, J., Bechstein, W.-O. and Woeste, G. (2012) Neoadjuvant Short- or Long-Term Radio(Chemo)- 
Therapy for Rectal Cancer: How and Who Should Be Treated? Digestive Disease, 30, 102-108. http://dx.doi.org/10.1159/000342038

[12] Kim, D.J. and Jung, K.H. (2006) Radiation Therapy for Rectal Cancer. The Korean Journal of Gastroenterology, 47, 285-290.

[13] Quirk, P., Steele, R., Monson, J., Grieve, R., Khanna, S., Couture, J., O’Callaghan, C., Myint, A.S., Bessell, E., Thompson, L.C., Parmar, M., Stephens, R.J. and Sebag-Montefiore, D. (2009) Effect of the Plane of Surgery Achieved on Local Recurrence in Patients with Operable Rectal Cancer: A Prospective Study Using Data from the MRC CR07 and NCIC-CTG CO16 Randomized Clinical Trial. Lancet, 373, 821-828. http://dx.doi.org/10.1016/S0140-6736(09)60485-2

[14] Sebag-Montefioreet, D., Stephens, R.J., Steele, R., Monson, J., Grieve, R., Khanna, S., Quirke, P., Couture, J., de Metz, C., Myint, A.S., Bessell, E., Griffiths, G., Thompson, L.C. and Parmar, M. (2009) Preoperative Radiotherapy versus Selective Postoperative Chemoradiotherapy in Patients with Rectal Cancer (MRC CR07 and NCIC-CTG C016): A Multicentre, Randomized Trial. Lancet, 373, 811-820. http://dx.doi.org/10.1016/S0140-6736(09)60484-0

[15] Garcia-Aguilar, J., Mellgren, A. and Rothenberger, D.A. (2000) Local Excision of Rectal Cancer without Adjuvant Therapy. Annals of Surgery, 231, 345-351. http://dx.doi.org/10.1097/00000658-200003000-00007

[16] Sengupta, S. and Tiandra, J.J. (2001) Local Excision of Rectal Cancer: What Is the Evidence? Diseases of the Colon \& Rectum, 44, 1345-1361. http://dx.doi.org/10.1007/BF02234796

[17] Phillip, P., Nash, G.M. and Wong, W.D. (2002) Long-Term Results of Local Excision for Rectal Cancer. Annals of Surgery, 236, 522-530. http://dx.doi.org/10.1097/00000658-200210000-00015

[18] Stipa, F., Lucandri, G., Ferri, M., Casula, G. and Ziparo, V. (2004) Local Excision of Rectal Cancer with Transanal Endoscopic Microsurgery (TEM). Anticancer Research, 24, 1167-1172.

[19] Suppiah, A., Hunter, I.A., Cowley, J., Garimella, V., Cast, J., Hartley, J.E. and Monson, J.R.T. (2009) Magnetic Resonance Imaging Accuracy in Assessing Tumor Down-Staging following Chemoradiation in Rectal Cancer. Colorectal Disease, 11, 249-253.

[20] Bebenek, M., Pudełko, M., Cisarż, K., Balcerzak, A., Tupikowski, W., Wojciechowski, L., Stankowska, A., Tarkowski, R. and Szulc, R. (2007) Therapeutic Results in Low-Rectal Cancer Patients Treated with Abdominosacral Resection Are Similar to Those Obtained by Means of Anterior Resection in Mid- and Upper-Rectal Cancer Cases. European Journal of Surgical Oncology, 33, 320-333. http://dx.doi.org/10.1016/j.ejso.2006.09.012

[21] Lezoch, E., Guerrieri, M., Paganini, A.M., D’Ambrosio, G., Baldarelli, M., Lezoche, G., Feliciotti, F. and De Sanctis, A. (2005) Transanal Endoscopic versus Total Mesorectal Laparoscopic Resections of $\mathrm{T}_{2}-\mathrm{N}_{0}$ Low Rectal Cancers after Neoadjuvant Treatment. Surgical Endoscopy, 19, 751-756. http://dx.doi.org/10.1007/s00464-004-8930-x

[22] Lezoch, G., Baldarelli, M., Guerrieri, M., Paganini, A.M., De Sanctis, A., Bartolacci, S. and Lezoche, E. (2008) A Prospective Randomized Study with a 5-Year Minimum Follow-Up Evaluation of Transanal Endoscopic Microsurgery versus Laparoscopic Total Mesorectal Excision after Neoadjuvant Therapy. Surgical Endoscopic, 22, 278. http://dx.doi.org/10.1007/s00464-007-9714-x

[23] Habr-Gama, A., Perez, R.O., Lynn, P.B., Neto, A.S. and Gama-Rodrigues, J. (2011) Nonoperative Management of Distal Rectal Cancer after Chemoradiation: Experience with the "Watch \& Wait" Protocol. In: Santoro, G.A., Ed., Rectal Cancer, a Multidisciplinary Approach to Management, InTech, Sao Paulo, Chapter 17. http://dx.doi.org/10.5772/1293

[24] Reis Neto, J.A., Quilici, F.A. and Reis, J.A. (1989) A Comparison of Nonoperative vs. Preoperative Radiotherapy in Rectal Carcinoma: A 10-Year Randomized Trial. Diseases of the Colon \& Rectum, 32, 702-710. http://dx.doi.org/10.1007/BF02555778

[25] Reis Neto, J.A., et al. (2000) Long-Term Results of Preoperative Radiotherapy for Cancer of the Lower Rectum. In: Reis Neto, J.A., Ed., New Trends in Coloproctology, Revinter, São Paulo, 401-410.

[26] Reis Neto, J.A., Quilici, F.A., Cordeiro, F., Ciquini, S. and Reis Jr., J.A. (1999) Pre-Operative Radiotherapy in Rectal Cancer: Evaluation of Irradiation Effects on Cellular Undifferentiation and Its Influence on Prognosis. Hepato-Gastroenterology, 46, 2825-2830.

[27] Reis Neto, J.A., et al. (2000) Preoperative Radiotherapy in Cancer of the Lower Rectum. Monduzzi, Colon, 67-74.

[28] Reis Neto, J.A., et al. (2001) Pre-Operative Radiotherapy and Laparoscopic Total Mesorectum Excision: Is There Any Improvement? Proktologia, 3, 141-142.

[29] Papillon, J. (1984) New Prospects in the Conservative Treatment of Rectal Cancer. Diseases of the Colon and Rectum, 27, 566-567. http://dx.doi.org/10.1007/BF02554589

[30] Papillon, J. (1980) Rectal and Anal Cancer. Springer-Verlag, Berlin, 24-32. 